erscheint letztlich weniger als „Notwehr“ denn als Übermut.

Jeder der Tagungen plant, organisiert und die Vorträge publiziert, wird mit dem intrinsischen Problem konfrontiert, aus der Vielfalt ein harmonisches Ganzes zu gestalten. Ein Projekt wie die deutsch-italienischen Expertengespräche verdoppelt gewissermaßen die Schwierigkeiten. Umso höher ist die Arbeit der Herausgeber und Tagungsorganisatoren zu schätzen. Dank ihrer kompetenten Referenten ist es ihnen gelungen, neue Einblicke zu verschaffen und neue Erkenntnisse zu vermitteln. Das Ergebnis sollte Ansporn sein, diese beispielhafte bilaterale Zusammenarbeit nicht nur sporadisch sondern konsequent fortzusetzen. Dies ist zugegebener Maßen ein großes Wort, gelassen ausgesprochen, denn eine Vollfinanzierung solcher Veranstaltungen, in diesem Fall dank der Deutschen Forschungsgemeinschaft, wird nicht die Regel sein. Selbst wenn sie auch nur ein kleiner - aber wichtiger - Mosaikstein im Rahmen einer europäischen Zusammenarbeit sind, wären sie dennoch ein Kapital „das geräuschlos unberechenbare Zinsen spendet“.

\section{Stephan Füssel, Ute Schneider (Hg.): Meilensteine buchwissenschaftlicher Forschung. Ein Reader zentraler Quellen und Materialien. Herausgegeben von Stephan Füssel und Ute Schneider in Zusammenarbeit mit einer Studierendengruppe. Wiesbaden: Harrassowitz, 2017. (Mainzer Studien zur Buchwissenschaft; Band 25). VI, 437 S., s/w. Abb., brosch. ISBN 978-3-447-10600-9, ISSN 0946-090X.€19,90}

Besprochen von Prof. Dr. Peter Vodosek: Seestraße 89, D-70174 Stuttgart, E-Mail: vodosek@hdm-stuttgart.de

https://doi.org/10.1515/bfp-2018-0059

Im Jahr 2014 haben Stephan Füssel und Corinna NorickRühl eine Einführung in die Buchwissenschaft veröffentlicht. Sie wollten mit dieser Publikation „bei der Wahl eines möglichen Studienfaches behilflich sein, beziehungsweise ganz konkret für Bachelorstudierende buchwissenschaftlicher Studiengänge Hintergrundinformationen bieten “. ${ }^{1}$

Mit der vorliegenden Neuerscheinung setzt die Mainzer Buchwissenschaft ihr vielversprechendes Unternehmen

1 Füssel, Stephan; Norick-Rühl, Corinna (2014): Einführung in die Buchwissenschaft. Darmstadt: WBG Wissenschaftliche Buchgesellschaft. Dazu die Rezension in: BIBLIOTHEK - Forschung und Praxis, 38 (2), 322-23. fort, Studierenden gediegene Lehrmaterialien in die Hand zu geben. Äußerer Anlass war das 70-jährige Bestehen der Buchwissenschaft als akademische Disziplin, die im Wintersemester 1947/48 als „Gutenberg-Lehrstuhl“ ins akademische Leben trat. Die Herausgeber Stephan Füssel und Ute Schneider deklarieren den umfänglichen Band als „Versuch, Studierenden der Buchwissenschaft anhand von impulsgebenden Texten eine gediegene Einführung in das Fach anzubieten und gleichzeitig Forschungsfragen in ihren historischen Kontext einzuordnen und zur weiterführenden Diskussion anzuregen." Das Format ist ein Reader, der 34 Grundlagentexte versammelt. Studierenden steht nunmehr auch in Deutschland ein Hilfsmittel zur Verfügung, das im angelsächsischen Bereich bereits Tradition hat, wenn man bei zwei Jahrzehnten bereits von Tradition sprechen will. Konkret gemeint ist der Book History Reader von David Finkelstein und Alistair McCleery vom schottischen Centre for the History of Book in Edinburgh, auf den auch an dieser Stelle nachdrücklich hingewiesen sei. ${ }^{2}$

Die Texte der Meilensteine werden in vier Blöcken zusammengefasst:

I. Quellen

II. Impulse für die Buchforschung

III. Theoretische Aspekte und Modelle

IV. Aktuelle Forschungsfragen

Die „Quellen“ beginnen mit dem Brief des Humanisten Enea Silvio Piccolomini von 1455, in welchem er von den ersten Lagen der 42-zeiligen Bibel berichtet. Exemplarisch seien noch genannt Texte von Gotthold Ephraim Lessing (1774/57), Philipp Erasmus Reich (1755), Giambattista Bodoni (1818) und weiter bis zu Samuel Fischer (1911) und Eugen Diederichs (1922). Von den „Impulsen“ seien die Autoren Robert Escarpit (1958/61), Rudolf Schenda (1970), Paul Raabe (1976) und Roger Chartier (1978/90) erwähnt. „Theoretische Aspekte“ bieten unter anderen Pierre Bourdieu (1999) und Robert Darnton (2007). Interessant ist ein Text von Aleida Assmann (2004), der obwohl im engeren Sinn nicht buchwissenschaftlich wegen seiner Bezugnahme auf mediale Umbruchsituationen aufgenommen wurde. Als Beispiele für „Aktuelle Forschungsfelder“ schließlich wurden zum Beispiel Texte von John P. Feather (1968), Robert Darnton (2008/2014) und John Bath/Scott Scholfield (2015), letztere wegen ihrer aktuellen Ausführungen zum „digital book“, aufgenommen.

Der Reader ist, wenn man so will, ein Gemeinschaftsprojekt der Herausgeber mit einer Gruppe von Masterstu-

2 Finkelstein, David; McCleery, Alistair (Ed.) (2006): The Book History Reader. London, New York: Routledge. 
dierenden. Zusammen trafen sie die Auswahl der Texte. Sie sind überwiegend in Deutsch, einige in Englisch sowie aus anderen Sprachen wie Latein in deutscher Übersetzung. Einige besitzen, wie die Herausgeber betonen, geradezu „kanonischen Charakter“, so dass ihre Berücksichtigung unumgänglich war. Über darüber hinausgehende Auswahlkriterien $\mathrm{zu}$ diskutieren, ist im gegebenen Fall müßig, zumal die Herausgeber die Meilensteine als „Gesprächsangebot und Arbeitsinstrument“ verstanden wissen wollen und der Diskurs in die Lehrveranstaltungen verlagert werden kann.

Den Texten, die von den beteiligten Studierenden verfasst wurden, sind Erläuterungen und Hinweise beigefügt, zumeist in vier Abschnitte gegliedert:

- Ein Stellenkommentar mit Begriffserklärungen

- Ein Kommentar zur Einordnung der Texte in den historischen Zusammenhang

- Verwendete Literatur

- Weiterführende Literatur

Das Projekt Meilensteine schließt die berühmte Lücke, wofür allen Beteiligten gleichermaßen zu danken ist. Das Studienbuch ist aber mehr als eines nur für die Buchwissenschaft. Es bietet eine interessante Lektüre für Fächer wie Sozialgeschichte, Literatursoziologie, Mediengeschichte und Kulturgeschichte allgemein. Generell ist aber hervorzuheben, dass die Mainzer Buchforschung bei aller Aktualität ihrer Themen den historischen Grundlagen die ihnen zukommende Bedeutung zumisst. Das ist nicht selbstverständlich. Deshalb soll zum Abschluss ein Exkurs folgen.

In der verwandten Bibliothekswissenschaft, die wie die Buchwissenschaft ein Kind der „Literärgeschichte“3 ${ }^{\text {ist, }}$ sind an den deutschen Universitäten mit einschlägigen Studienangeboten bibliothekshistorische Fragestellungen weitgehend obsolet geworden, obwohl es, was Reader betrifft, durchaus eine Traditionslinie gibt. Auch hier ist der angelsächsische Bereich vorangegangen. ${ }^{4}$ In der Bundesrepublik begann 1967, herausgegeben vom Deutschen Büchereiverband und vom Verein der Bibliothekare an Öffentlichen Büchereien, im Verlag Harrassowitz die Reihe B Quellen und Texte der Beiträge zum Büchereiwesen zu erscheinen. In sechs Bänden, die zwischen 1967 und 1978

3 Als Beispiel vergl. dazu den 1. Teil der „Einführung in die Bücherkunde“ von Denis, Michael (1777): Wien: Trattner.

4 Im Verlag NCR Microcard Editions, der seit 1969 die Reihe Reader Series in Library and Information Science herausgab, erschien 1971 von Michael M. Harris (Ed.) der „Reader in American Library History“ mit 24 wegweisenden Texten der (damals) letzten 25 Jahre, die lebhafte Diskussionen auslösten. publiziert wurden, wurden thematisch nach Epochen gegliedert Quellentexte zur Geschichte der Öffentlichen Bibliotheken vom 18. Jahrhundert bis 1961 ediert und kommentiert. ${ }^{5}$ Zwei in der chronologischen Abfolge fehlende Zeitabschnitte wurden 1984 und 1985 in zwei Bänden der Reihe Bibliothekswissenschaftliche Arbeiten aus dem Deutschen Bucharchiv München nachgetragen und ebenfalls bei Harrassowitz verlegt. Ende der 1970er-Jahre schlug der Verlag eine zweibändige Edition von Texten zur Geschichte der wissenschaftlichen Bibliotheken vor, die von Rupert Hacker (München) und vom Rezensenten erarbeitet werden sollte, die aber letztlich nicht zustande kam. Ob ein solches Projekt heutzutage noch zu realisieren wäre, erscheint fraglich.

Resümierend soll festgehalten werden, dass die $M e i-$ lensteine über das Umfeld der Studierenden der Buchwissenschaft hinaus allen kulturgeschichtlich Interessierten und Engagierten empfohlen werden können. Der erstaunlich günstige Preis der Paperback-Ausgabe sollte kein Hindernis sein. Der Rezensent schließt sich den Wünschen der Herausgeber nach ,aufgeschlossenen Lesern für weitere fruchtbringende Diskussionen über das leistungsstarke Medium Buch in einer lebendigen Buchwissenschaft“ an.

5 In den Beiträgen zum Büchereiwesen sind erschienen: Vodosek, Peter (Hrsg.) (1978): Vorformen der Öffentlichen Bibliothek, Mirbt, Karl-Wolfgang (Hrsg.) (1969): Pioniere des öffentlichen Bibliothekswesens, Thauer, Wolfgang (Hrsg.) (1970): Die Bücherhallenbewegung, Thauer, Wolfgang (Hrsg.) (1975): Politik der Bücherei. Paul Ladewig und die jüngere Bücherhallenbewegung, Joerden, Rudolf (Hrsg.) (1967): Zur Organisation des Städtischen Büchereiwesens, Andrae, Friedrich (1970): Volksbücherei und Nationalsozialismus (Materialien zur Theorie und Politik des öffentlichen Büchereiwesens in Deutschland 1933-1945). In der Reihe Buchwissenschaftliche Beiträge aus dem Deutschen Bucharchiv München wurden veröffentlicht: Vodosek, Peter (Hrsg.) (1985): Auf dem Weg zur öffentlichen Literaturversorgung. Quellen und Texte zur Geschichte der Volksbibliotheken in der zweiten Hälfte des 19. Jahrhunderts sowie Thauer, Wolfgang (Hrsg.) (1984): Die Öffentliche Bücherei der Weimarer Zeit. Quellen und Texte. 